\title{
Artificial neural network models for early diagnosis of hepatocellular carcinoma using serum levels of a-fetoprotein, a-fetoprotein-L3, des-y-carboxy prothrombin, and Golgi protein 73
}

\author{
Bo Li ${ }^{1}{ }^{1}$, Boan $\mathrm{Li}^{1, *}$, Tongsheng Guo ${ }^{1}$, Zhiqiang Sun ${ }^{1}$, Xiaohan $\mathbf{L i}^{1,2}$, Xiaoxi $\mathbf{L i}^{1}$, Lin \\ Chen ${ }^{1,2}$, Jing Zhao ${ }^{1}$ and Yuanli Mao ${ }^{1,2}$ \\ ${ }^{1}$ Center for Clinical Laboratory, 302 Millitary Hospital, Beijing, China \\ ${ }^{2}$ Graduate Student Team, Medical University of PLA, Beijing, China \\ *These authors contributed equally to this work \\ Correspondence to: Yuanli Mao, email: pipi780816@aliyun.com \\ Keywords: hepatocellular carcinoma, artificial neural network, serum tumor biomarker \\ Received: April 07, $2017 \quad$ Accepted: June 02, $2017 \quad$ Published: July 17, 2017 \\ Copyright: Li et al. This is an open-access article distributed under the terms of the Creative Commons Attribution License 3.0 \\ (CC BY 3.0), which permits unrestricted use, distribution, and reproduction in any medium, provided the original author and source \\ are credited.
}

\section{ABSTRACT}

More than $\mathbf{7 0} \%$ of hepatocellular carcinoma (HCC) cases develop as a consequence of liver cirrhosis (LC). Here we have evaluated the diagnostic potential of four serum biomarkers, and developed models for HCC diagnosis and differentiation from LC patients. Serum levels of a-fetoprotein (AFP), AFP-L3, des-y-carboxy prothrombin (DCP), and Golgi protein 73 (GP73) were analyzed in 114 advanced HCC patients, 81 early stage HCC patients, and 152 LC patients. Multilayer perceptron (MLP) and radial basis function (RBF) neural networks were used to construct the diagnostic models. Using all stages, HCC diagnostic models had a higher sensitivity ( $>70 \%)$ than the individual serum biomarkers, whereas only early stage HCC diagnostic models had a higher specificity ( $>80 \%$ ). The early stage HCC diagnostic models could not be used as HCC screening tools due to their low sensitivity (about $40 \%$ ). These results suggest that a combination of the two models might be used as a screening tool to distinguish early stage HCC patients from LC patients, thus improving prevention and treatment of HCC.

\section{INTRODUCTION}

Liver cancer is the sixth most common cancer throughout the world, but it is the third leading cause of cancer-related death due to its very poor prognosis. Hepatocellular carcinoma (HCC) is the major histological subtype of liver cancer. The major risk factors of HCC are infections with the hepatitis $\mathrm{B}$ and $\mathrm{C}$ viruses, which increase the risk of liver cancer by about 20-fold [1]. More than $90 \%$ of HCC cases develop as a consequence of underlying liver diseases, and liver cirrhosis (LC) occurs in $80 \%$ of HCC cases [2-4]. More than $60 \%$ of patients are diagnosed with late-stage disease after metastasis has occurred [5], resulting in an overall 5-year survival rate of $<16 \%$ [6]. However, if appropriate treatments are performed in early stages, the 5 -year survival rates of $\mathrm{HCC}$ patients exceed $75 \%$, highlighting the need to diagnose HCC at early stages in order to achieve the greatest possibility of curative treatment [7]. According to the American Association for the Study of Liver Diseases (AASLD) practice guidelines, curative treatment can be performed in the early stage of HCC (BCLC 0 -A), while in the advanced stages (BCLC B-D), only palliative or symptomatic treatments are available [8].

The AASLD guidelines also recommend that $\alpha$-fetoprotein (AFP) and ultrasound examination be used for HCC surveillance in hepatic cirrhosis population, but early stage $\mathrm{HCC}$ can be hardly differentiated from cirrhotic 
nodules because they have similar features on imaging [9-11]. AFP has been used as an HCC serum biomarker for many years, but its sensitivity is only about 39\%-65\% [12]. Several other tumor markers have been reported as good complements to AFP and have been used in clinical diagnosis, including lens culinaris agglutinin reactive AFP (AFP-L3), des- $\gamma$-carboxy prothrombin (DCP) and Golgi protein 73 (GP73) [13-18], but they do not meet the clinical requirements for early stage $\mathrm{HCC}$ diagnosis.

Artificial neural network (ANN) is a mathematical model that simulates the structure of biological neural networks. It possesses the characteristics of parallel information processing, distributed information storage, high non-linearity, good fault-tolerance and strong selflearning, self-organizing, and adaptive ability [19]. ANN has been widely applied in the fields of disease diagnosis and prediction [20-24]. The aim of this study was to develop effective HCC diagnostic models using ANN and four serum tumor biomarkers (AFP, AFP-L3, GP73, and DCP). These models can be used as a preliminary screening tool to distinguish early stage HCC patients from LC patients, thus improving prevention and treatment of HCC.

\section{RESULTS}

\section{Serum levels of AFP, AFP-L3, GP73, and DCP as HCC diagnostic markers}

347 HCC and LC patients were recruited and divided into three groups: 114 advanced HCC patients, 81 early stage HCC patients, and 152 LC patients. The demographic data of the patients are shown in Table 1. There were no significant differences in age, HBV infection rate, history of infection, and liver function indexes (bilirubin and alanine transaminase) among the three groups. However, there were significant differences in gender, serum albumin levels, and prothrombin time $(\mathrm{p}<0.05)$. The data indicated that male patients were at a higher risk to develop HCC, and LC patients had a worse liver synthesis function compared with HCC patients.

To evaluate the diagnostic value of the four serum tumor markers (AFP, AFP-L3, GP73, and DCP) in the progression of $\mathrm{HCC}$, we measured their concentrations in serum of all patients. The serum levels of all four markers differed $(\mathrm{p}<0.05)$ between early stage HCC patients and LC patients. AFP, AFP-L3, and DCP also showed significant changes between advanced HCC patients and LC patients $(\mathrm{p}<0.05$; Table 2$)$. The serum levels of AFP, AFP-L3, and DCP gradually increased during the progression of cirrhosis to HCC. However, the serum GP73 levels in LC patients were higher than in advanced and early stages HCC patients; the early stage HCC patients had the lowest serum levels of GP73. In addition, there was no significant difference between advanced HCC patients and LC patients $(p=0.112$; Figure 1). In order to determine whether GP73 could be used in the diagnosis of HCC, we compared serum GP73 levels in LC patients and patients with all stages of HCC. The levels of GP73 differed $(\mathrm{p}<0.001, \mathrm{Z}=-3.728)$ between all stages HCC patients and LC patients.

\section{Serum levels of AFP, AFP-L3, GP73, and DCP as $\mathrm{HCC}$ diagnostic markers in cirrhotic patients}

ROC analysis was used to determine whether the serum AFP, AFP-L3, GP73, and DCP levels are powerful to diagnose $\mathrm{HCC}$ in the cirrhotic population, as measured by the AUROC. The optimal cut-off values were determined with the maximum sum of sensitivity and
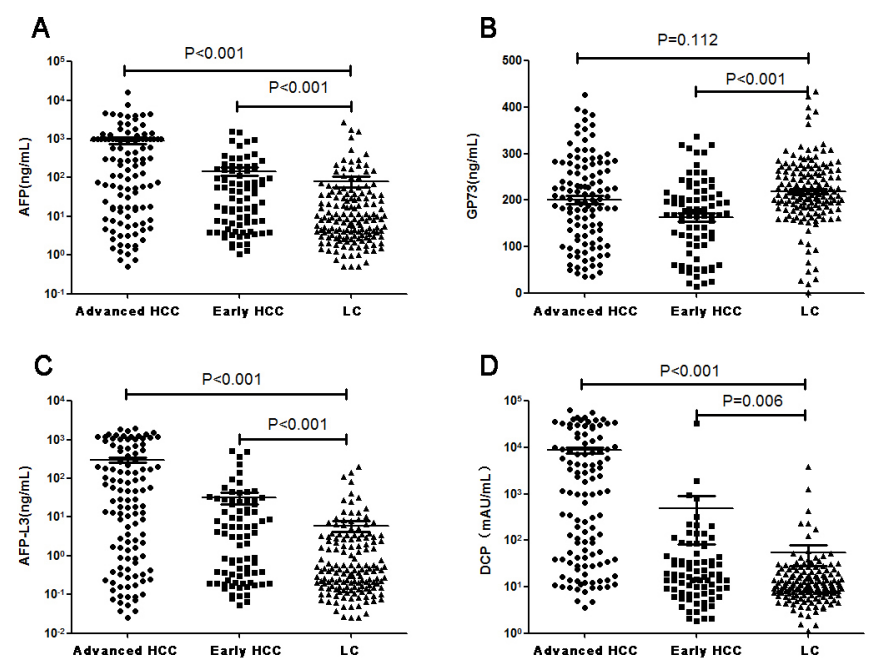

Figure 1: The difference in AFP (A), GP73 (B), AFP-L3 (C), and DCP (D) levels between advanced HCC, early stage HCC, and LC patients. 
Table 1: The demographic data of the patients

\begin{tabular}{lcccc}
\hline & Advanced HCC & Early HCC & LC & P value \\
\hline Male/ Female & $97 / 17$ & $65 / 16$ & $101 / 51$ & 0.001 \\
Age (yr) & $53(21-74)$ & $56(36-81)$ & $51(23-83)$ & 0.154 \\
HBV infection/others & $90 / 14$ & $69 / 12$ & $138 / 14$ & 0.377 \\
History of infection (yr) & $10.9 \pm 7.5$ & $14.1 \pm 6.5$ & $14.7 \pm 8.2$ & 0.183 \\
Albumin $(\mathrm{g} / \mathrm{L})$ & $33.4 \pm 6.0$ & $33.8 \pm 6.8$ & $30.7 \pm 6.3$ & 0.001 \\
Bilirubin $(\mu \mathrm{mol} / \mathrm{L})$ & $25.4(4.6-520.6)$ & $20.8(6.0-141.0)$ & $24.9(4.8-423.3)$ & 0.209 \\
Prothrombin time(s) & $13.3(10.7-22.2)$ & $13.1(10.6-21.9)$ & $13.9(10.2-23.2)$ & 0.001 \\
Alanine & $44(9-1194)$ & $39(7-263)$ & $42(10-1260)$ & 0.120 \\
transaminase(IU/L) & & &
\end{tabular}

Table 2: The statistical analysis of the levels of serum markers between three groups

\begin{tabular}{lccccccc}
\hline Markers & $\begin{array}{c}\text { Advanced HCC } \\
\text { Group }\end{array}$ & $\begin{array}{c}\text { Early HCC } \\
\text { Group }\end{array}$ & LC Group & \multicolumn{2}{c}{$\begin{array}{c}\text { Advanced HCC } \\
\text { vs LC }\end{array}$} & Early HCC vs LC \\
\cline { 5 - 8 } & & & & Z Value & P Value & Z Value & P Value \\
\hline AFP(ng/mL) & $224.0(0.5-16488.0)$ & $29.5(1.0-1574.0)$ & $8.4(0.5-2659.0)$ & -7.190 & $<0.001$ & -3.773 & $<0.001$ \\
GP73(ng/mL) & $202.2(35.4-427.4)$ & $168.5(13.1-337.5)$ & $214.0(1.6-434.5)$ & -1.591 & 0.112 & -5.103 & $<0.001$ \\
AFP-L3(ng/mL) & $26.96(0.03-1981.00)$ & $3.25(0.05-518.00)$ & $0.42(0.03-204.4)$ & -7.566 & $<0.001$ & -4.330 & $<0.001$ \\
DCP(mAU/mL) & $995.5(0.6-64091.0)$ & $16.3(0.2-33210.0)$ & $10.8(0.2-3778.4)$ & -10.426 & $<0.001$ & -2.736 & 0.006 \\
\hline
\end{tabular}
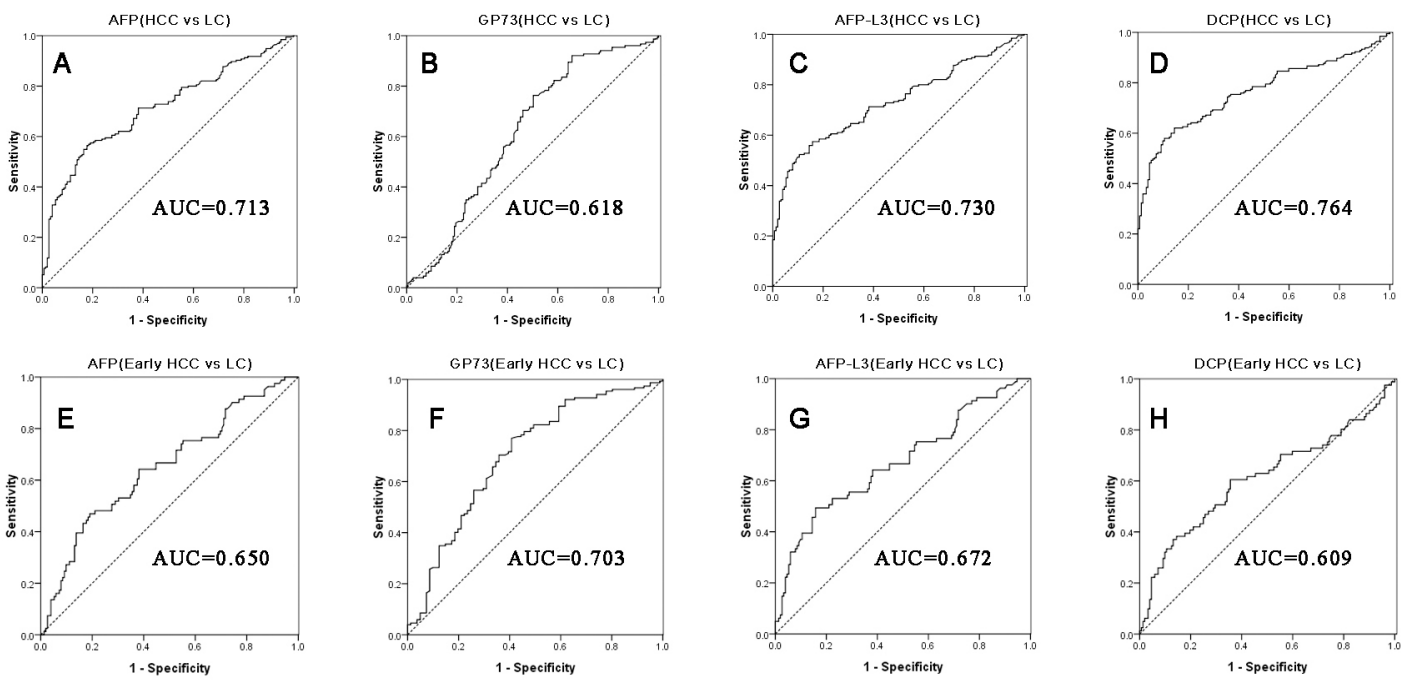

Figure 2: The ROC curves of AFP, GP73, AFP-L3, and DCP for diagnosis of all stages HCC and early stage HCC from the cirrhotic patients. Figures A-D demonstrate AFP, GP73, AFP-L3 and DCP diagnostic performance in all stages HCC, while Figures E-H are in early stage HCC. 
Table 3: The diagnostic performance of four markers and ANN models for all stages HCC and early stage HCC cases in cirrhotic patients

\begin{tabular}{|c|c|c|c|c|c|c|c|c|c|c|}
\hline \multirow[t]{2}{*}{ Markers } & \multicolumn{5}{|c|}{ HCC vs LC } & \multicolumn{5}{|c|}{ Early HCC vs LC } \\
\hline & AUC & p Value & Cut-off & Sensitivity & Specificity & AUC & p Value & Cut-off & Sensitivity & Specificity \\
\hline $\operatorname{AFP}(\mathrm{ng} / \mathrm{mL})$ & $0.713(0.659-0.767)$ & $<0.001$ & 49.4 & 0.564 & 0.822 & $0.650(0.575-0.725)$ & $<0.001$ & 43.7 & 0.469 & 0.809 \\
\hline GP73(ng/mL) & $0.618(0.559-0.677)$ & $<0.001$ & 148.6 & 0.921 & 0.344 & $0.703(0.630-0.776)$ & $<0.001$ & 181.4 & 0.770 & 0.593 \\
\hline AFP-L3(ng/mL) & $0.730(0.678-0.782)$ & $<0.001$ & 8.028 & 0.523 & 0.895 & $0.672(0.597-0.748)$ & $<0.001$ & 3.813 & 0.494 & 0.842 \\
\hline $\mathrm{DCP}(\mathrm{mAU} / \mathrm{mL})$ & $0.764(0.714-0.814)$ & $<0.001$ & 28.1 & 0.621 & 0.855 & $0.609(0.527-0.690)$ & 0.006 & 13.7 & 0.605 & 0.645 \\
\hline MLP-Models & $0.753(0.701-0.806)$ & $<0.001$ & 0.5 & 0.697 & 0.809 & $0.692(0.616-0.768)$ & $<0.001$ & 0.5 & 0.469 & 0.914 \\
\hline RBF-Models & $0.742(0.688-0.795)$ & $<0.001$ & 0.5 & 0.733 & 0.750 & $0.659(0.582-0.735)$ & $<0.001$ & 0.5 & 0.481 & 0.836 \\
\hline
\end{tabular}

Table 4: Diagnostic results of two models for all stages HCC and LC patients

\begin{tabular}{lccccccc}
\hline Group & Diagnosis & \multicolumn{3}{c}{ MLP Model } & \multicolumn{3}{c}{ RBF Model } \\
\cline { 3 - 7 } & & LC & HCC & Accuracy & LC & HCC & Accuracy \\
\hline \multirow{3}{*}{ Traning set } & LC & 82 & 17 & $82.8 \%$ & 76 & 29 & $72.4 \%$ \\
& HCC & 43 & 94 & $68.6 \%$ & 39 & 106 & $73.1 \%$ \\
& Total percentage & $53.0 \%$ & $47.0 \%$ & $74.6 \%$ & $46.0 \%$ & $54.0 \%$ & $72.8 \%$ \\
\multirow{3}{*}{ Test set } & LC & 25 & 10 & $71.4 \%$ & 23 & 6 & $79.3 \%$ \\
& HCC & 11 & 26 & $70.3 \%$ & 6 & 28 & $82.4 \%$ \\
& Total percentage & $50.0 \%$ & $50.0 \%$ & $70.8 \%$ & $46.0 \%$ & $54.0 \%$ & $81.0 \%$ \\
\multirow{3}{*}{ Holdout set } & LC & 16 & 2 & $88.9 \%$ & 15 & 3 & $83.3 \%$ \\
& HCC & 5 & 16 & $76.2 \%$ & 7 & 9 & $56.3 \%$ \\
& Total percentage & $53.8 \%$ & $46.2 \%$ & $82.1 \%$ & $64.7 \%$ & $35.3 \%$ & $70.6 \%$ \\
\hline
\end{tabular}

A

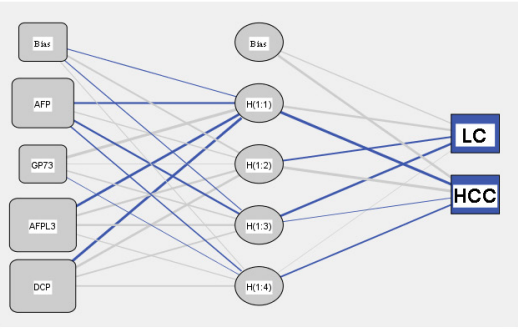

C

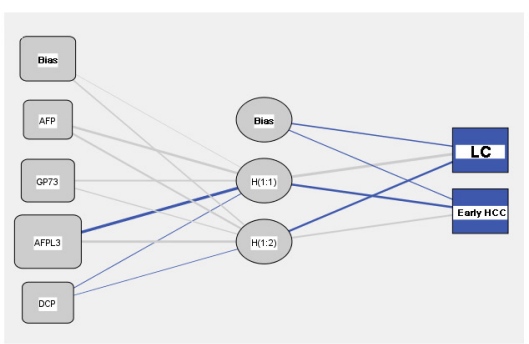

B

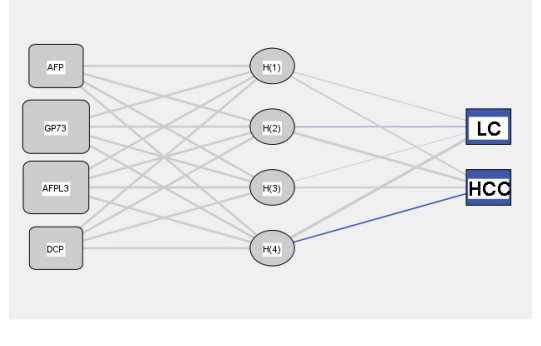

D

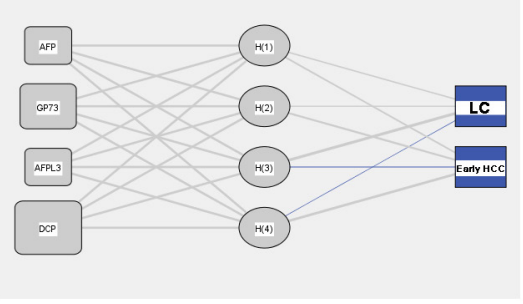

Figure 3: Architecture of neural network models. (A) MLP model for differentiating all stages HCC from LC patients; (B) RBF model for differentiating all stages HCC from LC patients; (C) MLP model for differentiating early stage HCC from LC patients; (D) RBF model for differentiating early stage HCC from LC patients. The blue lines represent synaptic weight $>1$, the grey lines represent synaptic weight $<1$. 
specificity. As shown in Figures 2A-2D, which illustrate diagnostic performance of the four serum markers to differentiate all stages of HCC patients (including early and advanced HCC) from LC patients, DCP levels achieved a better diagnostic performance than the levels of the other three markers; AUROC for DCP was 0.764 .
However, for the diagnosis of early stage HCC, GP73 levels demonstrated a better performance (AUC $=0.703$; Figures 2E-2H), indicating that GP73 has a superior early diagnostic ability than the other markers. As expected, AFP, AFP-L3, and DCP had a relatively better diagnostic performance for HCC than for early stage HCC. Table 3
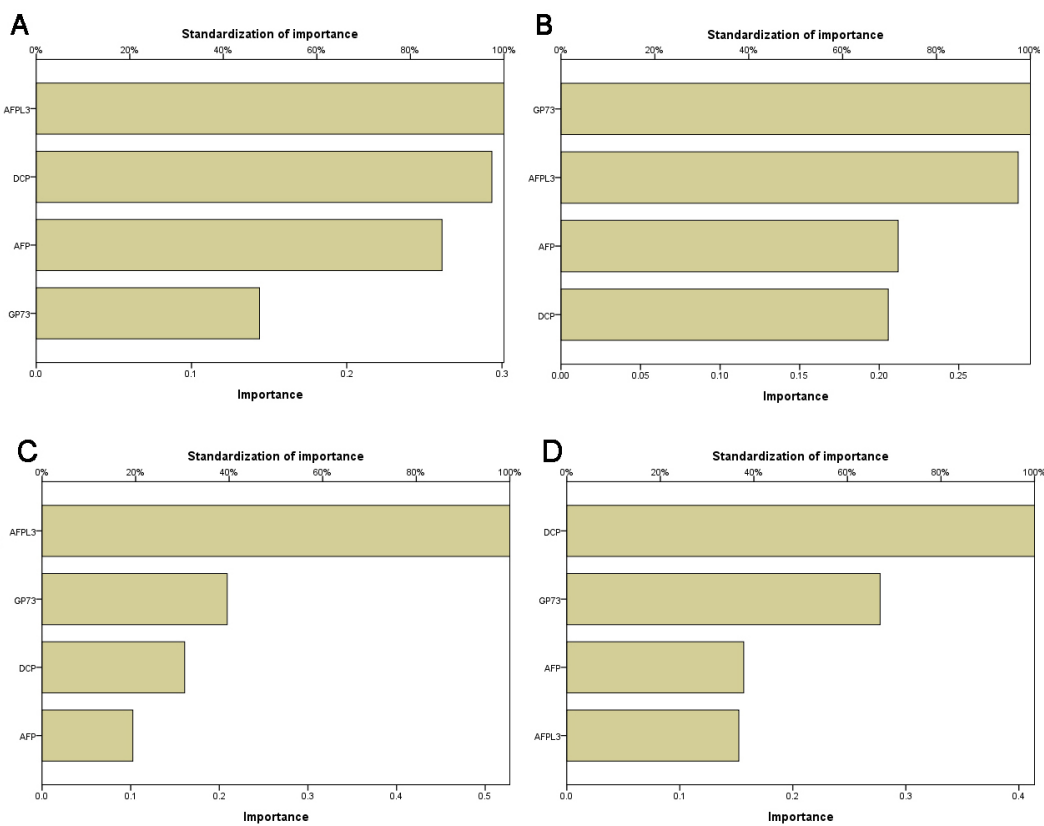

Figure 4: The relative importance of the four markers to the diagnostic models. (A) MLP model importance histogram for LC vs all stages HCC; (B) RBF model importance histogram for LC vs all stages HCC; (C) MLP model importance histogram for LC vs early stage HCC; (D) RBF model importance histogram for LC vs early stage HCC.
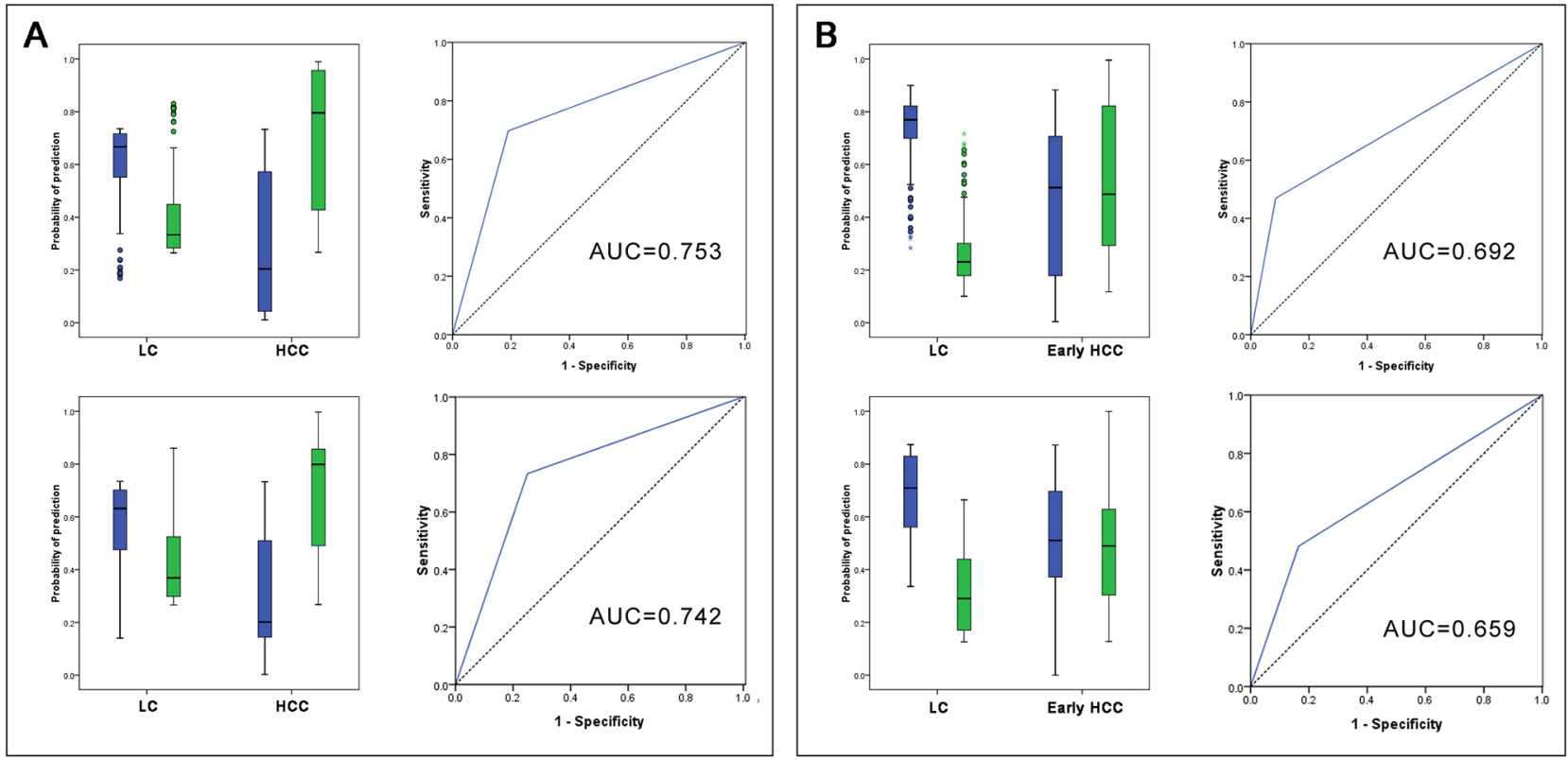

Figure 5: Prediction probability histograms and ROC curves for neural network models. (A) LC vs all stages HCC; (B) LC vs early stage HCC. The upload, MLP models; the download, RBF models. Green columns represent diagnosed HCC samples; blue columns represent diagnosed LC samples. 
shows that AFP-L3 has the best specificity and GP73 has the best sensitivity in early stage HCC, as well as in all stages of HCC.

\section{Development of neural network models to differentiate LC and HCC patients}

We used multilayer perceptron (MLP) and radial basis function (RBF) neural networks to construct the diagnostic models. Figure $3 \mathrm{~A}$ and $3 \mathrm{~B}$ show the architecture of two models; both of them included four input layers neurons and two output layers neurons. For the MLP model, $74.6 \%$ training samples, $70.8 \%$ testing samples, and $82.1 \%$ holdout samples were correctly diagnosed (Table 4). Analysis of the importance of the four serum markers showed that AFP-L3 was the most important variable in MLP model (100 \%); the following variables were DCP (97.4\%), AFP (86.8\%), and GP73 (47.7\%) (Figure 4A). For the RBF model, $72.8 \%$ training samples, $81.0 \%$ testing samples, and $70.6 \%$ holdout samples were correctly diagnosed (Table 4). GP73 was the most important variable, followed by AFP-L3 (97.4\%), AFP (71.8\%), and DCP (69.7\%) (Figure 4B). Prediction probability histograms (Figure 5A) showed that the MLP model could accurately recognize LC patients. In contrast, the RBF model had a better recognition ability for HCC patients. The AUROCs of MLP and RBF models were 0.753 and 0.742 in HCC diagnosis, respectively. These two models had a better diagnostic performance than the serum levels of AFP, AFP-L3, and GP73. Table 3 shows that the two models achieved a higher sensitivity than the individual serum biomarkers, even though their specificity was somewhat decreased.

\section{Development of neural network models to differentiate LC and early stage HCC patients}

Since prediction or early HCC diagnosis is more important than prediction of late-stage diagnosis, the main purpose of this study was to establish sensitive and accurate early stage HCC diagnostic models. We used MLP and RBF neural networks to construct the diagnostic models, using the early stage HCC and LC as the two model output layers neurons. As shown in Table 5 , the accuracies of the MLP model for training set, test set, and holdout set were $75.8 \%, 81.3 \%$, and $66.7 \%$, respectively. The order of importance was AFP-L3 (100\%), GP73 (39.6\%), DCP (30.5\%), and AFP (19.4\%) (Figure 4C). For the RBF model, the accuracies for the three sets were $71.1 \%, 70.0 \%$, and $75.0 \%$, respectively. The order of importance was DCP (100\%), GP73 (67.0\%), AFP (37.8\%), and AFP-L3 (36.8\%) (Figure 4D). Figure 5 illustrates that the two models could accurately identify LC patients, but could not identify early stage HCC patients. Their AUROCs were 0.692 and 0.659, respectively. However, in spite of the high specificities of both models, they had a lower sensitivity compared to the individual biomarkers (Table 3), indicating that they may increase the risk of missed HCC diagnosis.

\section{DISCUSSION}

In recent years, many promising candidate biomarkers for HCC have been identified, but most of them have not been applied in the clinical diagnosis due to their limited practicability and high cost [25-29]. Currently, AFP and imaging technology, such as ultrasound or computed tomography, are the two methods mainly used to diagnose HCC in hospitals. AFP has been used as an HCC serum biomarker for many years, but its sensitivity is only about $40 \%-65 \%$ [30]. AFP-L3, which is the main glycoform of AFP in the serum of HCC patients, has been proven to be an excellent biomarker with sensitivity of $75 \%$ to $97 \%$. High levels of AFP-L3 have been associated with poor differentiation, worse liver function, and larger tumor mass. Some studies have suggested that the AFP-L3/AFP ratio might be more helpful in diagnosis and prognosis of HCC than the AFP-L3 values [31, 32]. However, Miura and his coworkers have shown that the AFP-L3 levels cannot provide an entirely satisfactory solution to detect HCC at the early stage [33]. Our results show that the serum AFP-L3 levels gradually increase during the progression of cirrhosis to HCC. The AUROCs of AFP-L3 for HCC and early stage HCC were 0.730 and 0.672 , respectively. The serum AFP-L3 levels in early stage $\mathrm{HCC}$ patients (median $=3.25 \mathrm{ng} / \mathrm{mL}$ ) were higher than in LC patients (median $=0.42 \mathrm{ng} / \mathrm{mL}, \mathrm{p}<0.05$ ), suggesting that AFP-L3 may have a clinical value for the diagnosis of early HCC.

GP73 is a resident Golgi-specific membrane protein expressed by biliary epithelial cells in the liver. A metaanalysis study has suggested that GP73 is a valuable serum marker that seems to be superior to AFP and can be useful in the diagnosis and screening of HCC [34]. However, our results indicate that GP73 is elevated not only in $\mathrm{HCC}$, but also in LC; the concentration of GP73 in HCC (median=202.2 ng/mL) was lower than in LC patients (median=214.0 ng/mL, $\mathrm{p}<0.05$ ). The results of Tian et al. [35] are in agreement with our study; the median serum levels of GP73 were $107.3 \mu \mathrm{g} / \mathrm{L}$ in the HCC group and $141.2 \mu \mathrm{g} / \mathrm{L}$ in the LC group. Previous studies have shown that GP73 gene and protein levels gradually increase in chronic liver diseases; not only in hepatocytes, but also in activated hepatic stellate cells, which are the major cell type in liver cirrhosis [36-38]. Since we have observed maximal GP73 concentrations in liver cirrhosis rather than in HCC, these data suggest that GP73 might be regarded as a biomarker for liver cirrhosis rather than HCC. In addition, we have found that GP73 is the most sensitive biomarker to differentiate between early-stage $\mathrm{HCC}$ and LC patients; its sensitivity and AUROC are 0.770 and 0.703 , respectively. However, the low specificity of GP73 
Table 5: Diagnostic results of two models for early stage HCC and LC patients

\begin{tabular}{|c|c|c|c|c|c|c|c|}
\hline \multirow[t]{2}{*}{ Group } & \multirow[t]{2}{*}{ Diagnosis } & \multicolumn{3}{|c|}{ MLP Model } & \multicolumn{3}{|c|}{ RBF Model } \\
\hline & & LC & Early HCC & Accuracy & LC & Early HCC & Accuracy \\
\hline \multirow{3}{*}{ Traning set } & $\mathrm{LC}$ & 98 & 6 & $94.2 \%$ & 91 & 18 & $83.5 \%$ \\
\hline & Early HCC & 33 & 24 & $42.1 \%$ & 32 & 32 & $50.0 \%$ \\
\hline & $\begin{array}{c}\text { Total } \\
\text { percentage }\end{array}$ & $81.4 \%$ & $18.6 \%$ & $75.8 \%$ & $71.1 \%$ & $28.9 \%$ & $71.1 \%$ \\
\hline \multirow{3}{*}{ Test set } & $\mathrm{LC}$ & 29 & 5 & $85.3 \%$ & 23 & 5 & $82.1 \%$ \\
\hline & Early HCC & 4 & 10 & $71.4 \%$ & 7 & 5 & $41.7 \%$ \\
\hline & $\begin{array}{c}\text { Total } \\
\text { percentage }\end{array}$ & $68.8 \%$ & $31.3 \%$ & $81.3 \%$ & $75.0 \%$ & $25.0 \%$ & $70.0 \%$ \\
\hline \multirow{3}{*}{ Holdout set } & $\mathrm{LC}$ & 12 & 2 & $85.7 \%$ & 13 & 2 & $86.7 \%$ \\
\hline & Early HCC & 6 & 4 & $40.0 \%$ & 3 & 2 & $40.0 \%$ \\
\hline & $\begin{array}{c}\text { Total } \\
\text { percentage }\end{array}$ & $75.0 \%$ & $25.0 \%$ & $66.7 \%$ & $80.0 \%$ & $20.0 \%$ & $75.0 \%$ \\
\hline
\end{tabular}

limits its potential as an $\mathrm{HCC}$ biomarker, even though its sensitivity is extremely high.

DCP is an abnormal prothrombin molecule, which is induced by vitamin $\mathrm{K}$ absence II (PIVKAII), and may play an important role in promoting malignant HCC proliferation. Previous studies have shown that the serum DCP levels in patients with benign and malignant liver diseases are higher than in healthy people, suggesting that DCP might have a higher diagnostic sensitivity compared to AFP [39-42]. Our results show that the DCP levels in LC and all stages HCC patients have sensitivity of $62.1 \%$ and specificity of $85.5 \%$. For early stage HCC, the sensitivity and specificity of DCP are $60.5 \%$ and $64.5 \%$, respectively. DCP has been suggested as a biomarker for early stage $\mathrm{HCC}$ detection. Our results demonstrate that the DCP sensitivity is about $60 \%$ for $\mathrm{HCC}$ at an early stage. Although our study demonstrates that the sensitivity of DCP is higher than that of AFP in the diagnosis of early stage HCC, the DCP specificity is relatively low.

Artificial neural network (ANN) is ideal for diagnosis or prediction of disease in individuals, since it fits a nonlinear correlation between input and output variables [43-47]. There are various methods used for training of the network; MLP and RBF are the most common. Here, we have used these two networks to develop models for distinguishing early stage HCC from LC patients. Four serum biomarkers, AFP, AFP-L3, GP73, and DCP, have been used in the neural network modeling. The trained MLP and RBF models for recognition of all stages $\mathrm{HCC}$ and early stage $\mathrm{HCC}$ are presented in Tables 4 and 5. Single serum biomarkers are insufficiently precise for the diagnosis of $\mathrm{HCC}$, but using their combinations greatly increases the accuracy. The HCC diagnostic models that we have developed have excellent diagnostic potential: their accuracy exceeded
$80 \%$, and their sensitivity was improved compared to single biomarkers. However, the early-stage HCC diagnostic models have a relatively low sensitivity, which may lead to some missed diagnoses. Combination of both models should be a more reliable approach for the diagnosis of early-stage HCC. In the clinical practice, the HCC RBF model (sensitivity=73.3\%) might be used as a screening tool for detection of early stage $\mathrm{HCC}$ and its differentiation from LC, while the early stage MLP HCC model (specificity $=91.4 \%$ ) might be applied to exclude false positives. This strategy should not only improve $\mathrm{HCC}$ detection rates, but also reduce false positives in early HCC stages.

In conclusion, we have evaluated the potential of AFP, AFP-L3, GP73, and DCP serum biomarkers for HCC diagnosis, and developed diagnostic models using these biomarkers and MLP and RBF neural networks to differentiate HCC and early stage HCC from LC patients. These models can differentiate HCC and early stage HCC from liver cirrhosis. Future studies will be necessary to test their potential for clinical benefit in HCC patients.

\section{MATERIALS AND METHODS}

\section{Human subjects}

347 subjects were recruited from outpatients and inpatients of the 302 Military Hospital of China (114 advanced HCC patients, 81 early stage HCC patients, and 152 LC patients) from February 2013 to December 2015. The diagnosis of HCC was made by liver histopathology or MRI based on the guidelines from the ministry of health of the People's Republic of China [48]. The diagnosis of LC was based on clinical, laboratory and imaging evidence based on the guidelines from the 
Chinese Society of Hepatology and the Chinese Society of Infectious Diseases [49, 50]. The stage of tumor was based on BCLC staging system; patients with BCLC 0 -A stages were denoted as the early stage HCC group, and those with BCLC B-D stages were denoted as the advanced HCC group [8]. Patients with the following conditions were excluded: Patients with other systemic diseases, such as diabetes or hypertension, and patients with severe complications, such as upper gastrointestinal bleeding or hepatic encephalopathy. The study procedures were approved by the ethics committee of the 302 military hospital of China and written informed consent was obtained from all subjects.

\section{Laboratory tests}

The serum samples were collected in $5 \mathrm{~mL}$ vacuum blood collection tubes without anticoagulant, then centrifuged for $5 \mathrm{~min}$ at $12,000 \mathrm{~g}$ at room temperature. Serum concentrations of AFP and GP73 were measured using chemiluminescent immunoassay kits (Hotgen Biotech Co, China). For AFP-L3, the serum was first fractionated on lectin-affinity column, and Lens culinaris agglutinin selective elution was used to assay AFP-L3 by chemiluminescent immunoassay. Serum DCP levels were measured by Architect i2000 immunoassay analyzer (ARCHTECT PIVKA-II, Abbott Co, USA). Clinical tests were performed by an AU5400 automatic biochemical analyzer (Beckman Co, USA).

\section{Development of the neural network models}

Two types of ANN models, MLP and RBF, were developed by SPSS 17.0 Neural Network module. MLP and RBF are two popular architectures used in ANN; they are three-layer neural networks with input layer, hidden layer, and output layer. MLP is always trained by a backpropagation algorithm. When a neural group is provided with data through the input layer, the neurons in this first layer propagate the weighted data and randomly selected bias through the hidden layers. Once the net sum at a hidden node is determined, an output response is provided at the node using a transfer function. RBF neural network is a multilayer feed-forward network that can be used to identify nonlinear model effectively. The hidden layer transforms the data from the input space to the hidden space using a non-linear function. The output layer, which is linear, yields the response of the network [51].

In this study, four selected variables (AFP, GP73, AFP-L3, and DCP) were used as the input layer neurons, and two variables (LC and early stage $\mathrm{HCC}$ or all stages HCC) were used as the output layer neurons. All subjects were randomly divided into a training set, a test set and a holdout set at the ratio of 7:2:1. Training set is used to train the network, holdout set is used to assess model's performance, and test set is used to validate the results.

\section{Statistical analysis}

All statistical analyses were performed using the software SPSS 17.0. To assess the role of four tumor markers as diagnostic markers for $\mathrm{LC}$ or $\mathrm{HCC}$, receiver operating characteristic curves (ROC) were plotted, and the area under the curve (AUROC) was calculated. Data with normal distribution were analyzed with Student's $t$ tests or one-way analysis of variance; other data were analyzed by the Wilcoxon or Kruskal-Wallis tests.

\section{Author contributions}

Professor Boan Li conceived and initiated this project. All experiments in this paper were designed by Professor Yuanli Mao. Experiments were performed by Xiaohan Li, Xiaoxi Li, Lin Chen, and Jing Zhao. Bo Li, Tongsheng Guo, and Zhiqiang Sun analyzed the data, and generated the final figures and tables. Dr. Bo Li wrote the manuscript.

\section{ACKNOWLEDGMENTS}

This work was supported by the Capital Medical Development Project of China (2014-2-5031). The Capital Characteristic Clinical Application Research Project of China (Z171100001017180)

\section{CONFLICTS OF INTEREST}

There is no conflicts of interest.

\section{REFERENCES}

1. Parkin DM, Bray F, Ferlay J, Pisani P. Global cancer statistics, 2002. CA Cancer J Clin. 2005; 55:74-108.

2. Liovet JM, Burroughs A, Bruix J. Hepatocellular carcinoma. Lancet. 2003; 362:1907-1917.

3. Maddrey W. Hepatitis B: an important public health issue. J Med Virol. 2000; 61:362-366.

4. Zhang Z, Zhang Y, Wang Y, Xu L, Xu W. Alphafetoprotein-L3 and Golgi protein 73 may serve as candidate biomarkers for diagnosing alpha-fetoprotein-negative hepatocellular carcinoma. OncoTargets and Therapy. 2015; 9:123-129.

5. Altekruse SF, McGlynn KA, Reichman ME. Hepatocellular carcinoma incidence, mortality, and survival trends in the United States from 1975 to 2005. J Clin Oncol. 2009; 27:1485-1491.

6. Siegel R, Naishadham D, Jemal A. Cancer statistics. CA Cancer J Clin. 2013; 63:30.

7. Hernandez-Gea V, Turon F, Berzigotti A, Villanueva A. Management of small hepatocellular carcinoma in cirrhosis:Focus on portal hypertension. World J Gastroenterol. 2013; 19:1193-1199. 
8. Jordi B, Morris S. Management of Hepatocellular Carcinoma: An Update. Hepatology. 2011; 53:1020-1022.

9. Rhee H, Kim MJ, Park MS, Kim KA. Differentiation of early hepatocellular carcinoma from benign hepatocellular nodules on gadoxetic acid-enhanced MRI. Br J Radiol. 2012; 85:e837-844.

10. Suh YJ, Kim MJ, Choi JY, Park YN, Park MS, Kim KW. Differentiation of hepatic hyperintense lesions seen on gadoxetic acid-enhanced hepatobiliary phase MRI. AJR Am J Roentgenol. 2011; 197:W44-52.

11. Kumada T, Toyoda H, Tada T, Sone Y, Fujimori M, Ogawa $\mathrm{S}$, Ishikawa T. Evolution of hypointense hepatocellular nodules observed only in the hepatobiliary phase of gadoxetate disodium-enhanced MRI. AJR Am J Roentgenol. 2011; 197:58-63.

12. Daniele B, Bencivenga A, Megna AS, Tinessa V. Alpha fetoprotein and ultrasonography screening for hepatocellular carcinoma. Gastroenterology. 2004; 127:S108-112.

13. Liebman HA, Furie $\mathrm{BC}$, Tong MJ, Blanchard RA, Lo KJ, Lee SD, Coleman MS, Furie B. Des-gammacarboxy (abnormal) prothrombin as a serum marker of primary hepatocellular carcinoma. N Eng J Med. 1984; 310:1427-1431.

14. Nomura F, Ishijima M, Kuwa K, Tanaka N, Nakai T, Ohnishi K. Serumdes-gamma-carboxy prothrombin levels determined by a new generation of sensitive immunoassays in patients with small-sized hepatocellular carcinoma. Am J Gastroenterol. 1999; 94:650-654.

15. Aoyagi Y, Isemura M, Suzuki Y, Sekine C, Soga K, Ozaki T, Ichida F. Fucosylated alpha-fetoprotein as marker of early hepatocellular carcinoma. Lancet. 1985; 2:1353-1354.

16. Taketa K, Endo Y, Sekiya C, Tanikawa K, Koji T, Taga H, Satomura S, Matsuura S, Kawai T, Hirai H. A collaborative study for the evaluation of lectin-reactive alphafetoprotein in early detection of hepatocellular carcinoma. Cancer Res. 1993; 53:5419-5423.

17. Marrero JA, Romano PR, Nikolaeva O, Steel L, Mehta A, Fimmel CJ, Comunale MA, D'Amelio A, Lok AS, Block TM. GP73, a resident Golgi glycoprotein, is a novel serum marker for hepatocellular carcinoma. J Hepatol. 2005; 43:1007-1012.

18. Hu JS, Wu DW, Liang S, Miao XY. GP73, a resident Golgi glycoprotein, is sensibility and specificity for hepatocellular carcinoma of diagnosis in a hepatitis B-endemic Asian population. Med Oncol. 2009; 27:339-345.

19. Grossi E, Buscema M. Introduction to artificial neural networks. Eur J Gastroenterol Hepatol. 2007;19:1046-1054.

20. Ramesh AN, Kambhampati C, Monson JR, Drew PJ. Artificial intelligence in medicine. Ann R Coll Surg Engl. 2004; 86:334-338.

21. Tang J, Wu L, Huang H, Feng J, Yuan Y, Zhou Y, Huang P, $\mathrm{Xu}$ Y, Yu C. Back propagation artificial neural network for community Alzheimer's disease screening in China. Neural Regen Res. 2013; 8:270-276.
22. LaFaro RJ, Pothula S, Kubal KP, Inchiosa ME, Pothula VM, Yuan SC, Maerz DA, Montes L, Oleszkiewicz SM, Yusupov A, Perline R, Inchiosa MA Jr. Neural Network Prediction of ICU Length of Stay Following Cardiac Surgery Based on Pre-Incision Variables. PLoS One. 2015; 10:e0145395.

23. Asadi H, Dowling R, Yan B, Mitchell P. Machine learning for outcome prediction of acute ischemic stroke post intraarterial therapy. PLoS One. 2014; 9:e88225.

24. Li H, Luo M, Zheng J, Luo J, Zeng R, Feng N, Du Q, Fang $J$. An artificial neural network prediction model of congenital heart disease based on risk factors: A hospital-based casecontrol study. Medicine (Baltimore). 2017; 96:e6090.

25. Shirakawa H, Suzuki H, Shimomura M, Kojima M, Gotohda N, Takahashi S, Nakagohri T, Konishi M, Kobayashi N, Kinoshita T, Nakatsura T. Glypican-3 expression is correlated with poor prognosis in hepatocellular carcinoma. Cancer Sci. 2009; 100:1403-1407.

26. Riener MO, Stenner F, Liewen H, Soll C, Breitenstein S, Pestalozzi BC, Samaras P, Probst-Hensch N, Hellerbrand C, Müllhaupt B, Clavien PA, Bahra M, Neuhaus P, et al. Golgi phosphoprotein2 (GOLPH2) expression in liver tumors and its value as a serum marker in hepatocellular carcinomas. Hepatology. 2009; 49:1602-1609.

27. Osada S, Kanematsu M, Imai H, Goshima S. Clinical significance of Serum HGF and c-Met expression in tumor tissue for evaluation of properties and treatment of hepatocellular carcinoma. Hepatogastroenterology. 2008; 55:544-549.

28. Cheung ST, Fan ST, Lee YT, Chow JP, Ng IO, Fong DY, Lo CM. Albumin mRNA in plasma predicts post-transplant recurrence of patients with hepatocellular carcinoma. Transplantation. 2008; 85:81-87.

29. Ferracin M, Veronese A, Negrini M. Micromarkers: MiRNAs in cancer diagnosis and prognosis. Expert Rev Mol Diagn. 2010; 10:297-308.

30. Li B, Li B, Guo T, Sun Z, Li X, Li X, Wang H, Chen W, Chen P, Mao Y. The Clinical Values of Serum Markers in the Early Prediction of Hepatocellular Carcinoma. Biomed Res Int. 2017:5358615.

31. Gomaa AI, Khan SA, Leen EL, Waked I, Taylor-Robinson SD. Diagnosis of hepatocellular carcinoma. World J Gastroenterol. 2009; 15:1301-1314.

32. Khien VV, Mao HV, Chinh TT, Ha PT, Bang MH, Lac BV, Hop TV, Tuan NA, Don LV, Taketa K, Satomura S. Clinical evaluation of lentil lectin-reactive alpha-fetoprotein-L3 in histology proven hepatocellular carcinoma. Int J Bio Markers. 2001; 16:105-111.

33. Miura N, Osaki Y, Nagashima M, Kohno M, Yorozu K, Shomori K, Kanbe T, Oyama K, Kishimoto Y, Maruyama S, Noma E, Horie Y, Kudo M, et al. A novel biomarker TERTmRNA is applicable for early detection of hepatoma. BMC Gastroenterology. 2010; 10:46. https://doi.org/ 10.1186/1471-230X-10-46. 
34. Witjes CD, van Aalten SM, Steyerberg EW, Borsboom GJ, de Man RA, Verhoef C, Ijzermans JN. Recently introduced biomarkers for screening of hepatocellular carcinoma: a systematic review and meta-analysis. Hepatol Int. 2013; 7:59-64.

35. Tian L, Wang Y, Xu D, Gui J, Jia X, Tong H, Wen X, Dong Z, Tian Y. Serological AFP/Golgi protein 73 could be a new diagnostic parameter of hepatic diseases. Int J Cancer. 2011; 129:1923-1931.

36. Kladney RD, Bulla GA, Guo L, Mason AL, Tollefson AE, Simon DJ, Koutoubi Z, Fimmel CJ. GP73, a novel Golgilocalized protein upregulated by viral infection. Gene. 2000; 249:53-65.

37. Iftikhar R, Kladney RD, Havlioglu N, Schmitt-Gräff A, Gusmirovic I, Solomon H, Luxon BA, Bacon BR, Fimmel CJ. Disease- and cell-specific expression of GP73 in human liver disease. Am J Gastroenterol. 2004; 99:1087-1095.

38. Kladney RD, Cui X, Bulla GA, Brunt EM, Fimmel CJ. Expression of GP73, a resident Golgi membrane protein, in viral and nonviral liver disease. Hepatology. 2002; 35:1431-1440.

39. Carr BI, Kanke F, Wise M, Satomura S. Clinical evaluation of lens culinaris agglutinin-reactive alpha-fetoprotein and des-gammacarboxy prothrombin in histologically proven hepatocellular carcinoma in the United States. Dig Dis Sci. 2007; 52:776-782.

40. Volk ML, Hernandez JC, Su GL, Lok AS, Marrero JA. Risk factors for hepatocellular carcinoma may impair the performance of biomarkers: a comparison of AFP, DCP, and AFP-L3. Cancer Biomark. 2007; 3:79-87.

41. Bertino G, Neri S, Bruno CM, Ardiri AM, Calvagno GS, Malaguarnera M, Toro A, Malaguarnera M, Clementi S, Bertino N, Di Carlo I. Diagnostic and prognostic value of alpha-fetoprotein, des- $\gamma$-carboxy prothrombin and squamous cell carcinoma antigen immunoglobulin $\mathrm{M}$ complexes in hepatocellular carcinoma. Minerva Med. 2011; 102:363-371.

42. Lok AS, Sterling RK, Everhart JE, Wright EC, Hoefs JC, Di Bisceglie AM, Morgan TR, Kim HY, Lee WM, Bonkovsky HL, Dienstag JL. Des-gamma-carboxy prothrombin and alphafetoprotein as biomarkers for the early detection of hepatocellular carcinoma. Gastroenterology. 2010; 138:493-502.

43. Huang S, Xu Y, Yue L, Wei S, Liu L, Gan X, Zhou S, Nie S. Evaluating the risk of hypertension using an artificial neural network method in rural residents over the age of 35 years in a Chinese area. Hypertens Res. 2010; 33:722-726.

44. Tang ZH, Liu J, Zeng F, Li Z, Yu X, Zhou L. Comparison of prediction model for cardiovascular autonomic dysfunction using artificial neural network and logistic regression analysis. PLoS One. 2013; 8:e70571.

45. Harrison RF, Kennedy RL. Artificial neural network models for prediction of acute coronary syndromes using clinical data from the time of presentation. Ann Emerg Med. 2005; 46:431-439.

46. Colak MC, Colak C, Kocatürk H, Sağiroğlu S, Barutçu I. Predicting coronary artery disease using different artificial neural network models. Anadolu Kardiyol Derg. 2008; $8: 249-254$.

47. Hirose H, Takayama T, Hozawa S, Hibi T, Saito I. Prediction of metabolic syndrome using artificial neural network system based on clinical data including insulin resistance index and serum adiponectin. Comput Biol Med. 2011; 41:1051-1056.

48. Guidelines for diagnosis and treatment of primary hepatocellular carcinoma. Chinese Clinical Oncology. 2011; 16:929-946.

49. The guidelines of prevention and treatment for chronic hepatitis B (2010 version). Chinese journal of experimental and clinical infectious disease (Electronic Version). 2011; 5:79-100.

50. Hepatotogy Branch, Infectious and Parasitology branch, Chinese Medical Association. Guideline of prevention and treatment of hepatitis C. [Article in Chinese]. Zhonghua $\mathrm{Yu}$ Fang Yi Xue Za Zhi. 2004; 38:210-215.

51. Memarian H, Balasundram SK. Comparison between MultiLayer Perceptron and Radial Basis Function Networks for Sediment Load Estimation in a Tropical Watershed. J Water Res Prot. 2012; 4:870-876. 